\title{
Das Mathematikangstinterview (MAI): Erste psychometrische Gütekriterien
}

Kohn, Juliane ; Richtmann, Verena ; Rauscher, Larissa ; Kucian, Karin ; Käser, Tanja ; Grond, Ursina ; Esser, Günther ; von Aster, Michael

\begin{abstract}
Aktuelle Studienergebnisse verdeutlichen, dass sich Kinder bereits im frühen Grundschulalter hinsichtlich ihrer Mathematikangst unterscheiden und dass diese mit deren Mathematikleistung korreliert (Vukovic et al., 2013; Wu et al., 2012). Um eine valide Erfassung der Mathematikangst auch im deutschen Sprachraum zu ermöglichen, zielt diese Studie auf die Entwicklung und psychometrische Überprüfung eines kindgerechten und ökonomischen Verfahrens zur Erfassung der Mathematikangst im frühen Grundschulalter ab. Das Mathematikangstinterview (MAI) erfasst die Angstintensität sowie deren Auswirkung auf verschiedenen Angstebenen. Dazu werden rechenspezifische Situationen sprachlich und bildlich präsentiert und die Kinder u. a. gebeten, ihre Angst mittels Angstthermometer einzuschätzen. Die vorliegende Überprüfung der Gütekriterien erfolgte an einer Stichprobe von 136 Kindern im Alter von sieben bis zehn Jahren. Neben arithmetischen Operationen und Textaufgaben wurde die Selbsteinschätzung hinsichtlich der allgemeinen Ängstlichkeit, der Schulund Leistungsangst sowie der Selbstbewertung und Einstellung zu Mathematik erfragt. Die Ergebnisse verdeutlichen, dass das Interview eine hohe Reliabilität, ermittelt über die interne Konsistenz, aufweist. Zudem spiegeln die Ergebnisse zur Validität bisherige Untersuchungsergebnisse zur Mathematikangst wider und belegen eine zufriedenstellende Kriteriumsvalidität des MAI. Die moderaten Zusammenhänge zwischen Mathematikangst und Rechenleistung sind weder durch die kognitive Leistung noch durch die erfassten Ängste zu erklären. Durch das MAI fanden sich querschnittlich frühe Unterschiede in der Ausprägung der Mathematikangst sowie moderate Zusammenhänge mit der Rechenleistung. Weiterführend sind Längsschnittstudien zur Entwicklung der Mathematikangst sowie deren Wechselwirkung mit der Rechenleistung nötig, um einen Präventions- und Interventionsbedarf abzuleiten.
\end{abstract}

DOI: https://doi.org/10.1024/2235-0977/a000040

Posted at the Zurich Open Repository and Archive, University of Zurich

ZORA URL: https://doi.org/10.5167/uzh-79704

Journal Article

Accepted Version

Originally published at:

Kohn, Juliane; Richtmann, Verena; Rauscher, Larissa; Kucian, Karin; Käser, Tanja; Grond, Ursina; Esser, Günther; von Aster, Michael (2013). Das Mathematikangstinterview (MAI): Erste psychometrische Gütekriterien. Lernen und Lernstörungen, 2(3):177-189.

DOI: https://doi.org/10.1024/2235-0977/a000040 


\section{Lernen und Lernstörungen}

\section{Das Mathematikangstinterview(MAI): Erste psychometrische Gütekriterien --Manuskript-Entwurf--}

\begin{tabular}{|c|c|}
\hline Manuskriptnummer: & LLS-D-13-00012R1 \\
\hline Artikeltyp: & Empirische Arbeit \\
\hline Bereich/Kategorie: & Emotion \\
\hline Schlüsselwörter: & $\begin{array}{l}\text { Mathematikangst; Gütekriterien; klinische Diagnostik; Rechenleistungen; } \\
\text { Grundschulkinder }\end{array}$ \\
\hline Korrespond. Autor: & $\begin{array}{l}\text { Juliane Kohn, Dipl.-Psych. } \\
\text { Universität Potsdam, Exzellenzbereich Kognitionswissenschaften, Department } \\
\text { Klinische Psychology/Psychotherapie } \\
\text { Potsdam, GERMANY }\end{array}$ \\
\hline Erstautor: & Juliane Kohn, Dipl.-Psych. \\
\hline \multirow[t]{8}{*}{ Reihenfolge der Autoren: } & Juliane Kohn, Dipl.-Psych. \\
\hline & Verena Richtmann, Dipl.-Psych. \\
\hline & Larissa Rauscher, Dipl.-Psych. \\
\hline & Karin Kucian, Dr. \\
\hline & Tanja Käser, Dipl. Ing. Inf. \\
\hline & Ursina Grond, Lic. Phil. \\
\hline & Günter Esser, Prof. Dr. \\
\hline & Michael von Aster, Prof. Dr. \\
\hline Zusammenfassung: & $\begin{array}{l}\text { Aktuelle Studienergebnisse verdeutlichen, dass sich Kinder bereits im frühen } \\
\text { Grundschulalter hinsichtlich ihrer Mathematikangst unterscheiden und dass diese mit } \\
\text { deren Mathematikleistung korreliert (Vukovic et al., 2009; Wu et al., 2012). Um eine } \\
\text { valide Erfassung der Mathematikangst auch im deutschen Sprachraum zu } \\
\text { ermöglichen, zielt diese Studie auf die Entwicklung und psychometrische Überprüfung } \\
\text { eines kindgerechten und ökonomischen Verfahrens zur Erfassung der } \\
\text { Mathematikangst im frühen Grundschulalter ab. } \\
\text { Das Mathematikangstinterview (MAI) erfasst die Angstintensität sowie deren } \\
\text { Auswirkung auf verschiedenen Angstebenen. Dazu werden rechenspezifische } \\
\text { Situationen sprachlich und bildlich präsentiert und die Kinder u.a. gebeten, ihre Angst } \\
\text { mittels Angstthermometer einzuschätzen. } \\
\text { Die vorliegende Überprüfung der Gütekriterien erfolgte an einer Stichprobe von } 136 \\
\text { Kindern im Alter von sieben bis zehn Jahren. Neben arithmetischen Operationen und } \\
\text { Textaufgaben wurde die Selbsteinschätzung hinsichtlich der allgemeinen Ängstlichkeit, } \\
\text { der Schul- und Leistungsangst sowie der Selbstbewertung und Einstellung zu } \\
\text { Mathematik erfragt. } \\
\text { Die Ergebnisse verdeutlichen, dass das Interview eine hohe Reliabilität, ermittelt über } \\
\text { die interne Konsistenz, aufweist. Zudem spiegeln die Ergebnisse zur Validität bisherige } \\
\text { Untersuchungsergebnisse zur Mathematikangst wider und belegen eine } \\
\text { zufriedenstellende Kriteriumsvalidität des MAl. Die moderaten Zusammenhänge } \\
\text { zwischen Mathematikangst und Rechenleistung sind weder durch die kognitive } \\
\text { Leistung noch durch die erfassten Ängste zu erklären. } \\
\text { Durch das MAl fanden sich querschnittlich frühe Unterschiede in der Ausprägung der } \\
\text { Mathematikangst sowie moderate Zusammenhänge mit der Rechenleistung. } \\
\text { Weiterführend sind Längsschnittstudien zur Entwicklung der Mathematikangst sowie } \\
\text { deren Wechselwirkung mit der Rechenleistung nötig, um einen Präventions- und } \\
\text { Interventionsbedarf abzuleiten. }\end{array}$ \\
\hline
\end{tabular}


Mathematikangstinterview (MAI)

\section{Titelseite}

Das Mathematikangstinterview (MAI): Erste psychometrische Gütekriterien 


\section{Zusammenfassung (deutsch)}

Aktuelle Studienergebnisse verdeutlichen, dass sich Kinder bereits im frühen Grundschulalter hinsichtlich ihrer Mathematikangst unterscheiden und dass diese mit deren Mathematikleistung korreliert (Vukovic et al., 2009; Wu et al., 2012). Um eine valide Erfassung der Mathematikangst auch im deutschen Sprachraum zu ermöglichen, zielt diese Studie auf die Entwicklung und psychometrische Überprüfung eines kindgerechten und ökonomischen Verfahrens zur Erfassung der Mathematikangst im frühen Grundschulalter ab.

Das Mathematikangstinterview (MAI) erfasst die Angstintensität sowie deren Auswirkung auf verschiedenen Angstebenen. Dazu werden rechenspezifische Situationen sprachlich und bildlich präsentiert und die Kinder u.a. gebeten, ihre Angst mittels Angstthermometer einzuschätzen.

Die vorliegende Überprüfung der Gütekriterien erfolgte an einer Stichprobe von 136 Kindern im Alter von sieben bis zehn Jahren. Neben arithmetischen Operationen und Textaufgaben wurde die Selbsteinschätzung hinsichtlich der allgemeinen Ängstlichkeit, der Schul- und Leistungsangst sowie der Selbstbewertung und Einstellung zu Mathematik erfragt.

Die Ergebnisse verdeutlichen, dass das Interview eine hohe Reliabilität, ermittelt über die interne Konsistenz, aufweist. Zudem spiegeln die Ergebnisse zur Validität bisherige Untersuchungsergebnisse zur Mathematikangst wider und belegen eine zufriedenstellende Kriteriumsvalidität des MAI. Die moderaten Zusammenhänge zwischen Mathematikangst und Rechenleistung sind weder durch die kognitive Leistung noch durch die erfassten Ängste zu erklären.

Durch das MAI fanden sich querschnittlich frühe Unterschiede in der Ausprägung der Mathematikangst sowie moderate Zusammenhänge mit der Rechenleistung. Weiterführend sind Längsschnittstudien zur Entwicklung der Mathematikangst sowie deren Wechselwirkung mit der Rechenleistung nötig, um einen Präventions- und Interventionsbedarf abzuleiten.

Stichworte: Mathematikangst, Gütekriterien, klinische Diagnostik, Rechenleistungen, Grundschulkinder 


\section{Zusammenfassung (englisch)}

\section{Math Anxiety Interview (MAI): First psychometric properties}

Background: Math anxiety can be defined as ,...feelings of tension and anxiety that interfere with the manipulation of numbers and the solving of mathematical problems in a wide variety of ordinary life and academic situations" (Richardson \& Suinn, 1972, p. 551). Furthermore math anxiety meets the criteria for a specific phobia, which is characterised by physiological reactions, negative and dysfunctional cognitions and avoidance behaviour (Hembree, 1990; Lang, 1968). Of particular relevance is the avoidance behaviour, which has a wide influence on individual's math achievement, the engagement in math class and future selection of math related subjects (Ashcraft \& Kirk, 2001; Hembree, 1990). As a consequence, this might ultimately contribute to school phobia or psychosomatic distress (Ashcraft, 2002; Dowker, 2005). Devine, Fawcett, Szücs and Dowker (2012) specify a vicious circle in which math anxiety leads to negative experiences with math, which results in failure that strengthens in turn math anxiety and causes poor school performance (see also Dowker, Bennett \& Smith, 2012; Krinzinger \& Kaufmann, 2006). Math anxiety is associated with negative attitudes towards mathematics, less enjoyment of mathematics and a lower self-confidence in the subject (Ashcraft \& Kirk, 2001; Hembree, 1990; Krinzinger et al., 2007). Especially the relationship between math anxiety and math performance is well reviewed. Numerous studies showed a negative relationship between math anxiety and math performance, most notably for secondary school (Hembree, 1990; Ma, 1999). As a mechanism of action, the influence of math anxiety on working memory has been discussed (e.g. Ashcraft \& Kirk, 2001).

The majority of the findings concerning math anxiety are based on studies that refer to young adults (e.g. Ashcraft, Krause \& Hopko, 2007; Hopko, Mahadevan, Bare \& Hunt, 2003) or children that attend middle school (Meece, Wigfield \& Eccles, 1990). Over the past years the focus was expanded on children of early primary school age (Krinzinger, Kaufmann \& Willmes, 2009; Vukovic, Kieffer, Bailey \& Harari, 2013; Wu, Barth, Amin, Malcarne \& Menon, 2012). Studies showed different levels of math anxiety in young children and the relationship between 
math anxiety and math performance was demonstrated already for children in second and third grade (Ramirez, Gunderson, Levine \& Beilock, in press; Vukovic et al., 2009).

At this point of time there is one German questionnaire assessing math anxiety available for primary school children (class 1-3), that was developed by Krinzinger and colleagues (2007). The „Fragebogen für Rechenangst“ (FRA) is based on the „Math Anxiety Questionnaire“ (MAQ; Thomas \& Dowker, 2000). The authors reported only minor negative correlations between math anxiety scores and math performance (Krinzinger et al., 2007). Furthermore, longitudinal analyses showed no influence of math anxiety on the later math performance or vice versa (Krinzinger et al., 2009). Krinzinger and colleagues (2009) assume that the phrasing of the items in the questionnaire might be too difficult to be understood by young children.

Aims: The aim of the present study was the development of a new economical math anxiety measure suitable for children since there does not exist a valid instrument for the measurement of math anxiety for German-speaking early primary school children at this time (e.g. Krinzinger et al., 2009). Furthermore, psychometric properties of reliability and criterion validity are aimed to be determined. It is expected that a significant negative correlation between math anxiety and math performance can be detected.

Methods: The "Mathematikangstinterview" (MAI) combines two different types of questions while a math related situation is verbally and pictorially presented. The child is initially asked to rate its anxiety intensity concerning the presented situation by an anxiety thermometer. In a second step the different components of anxiety (affective, cognitive, behavioural and physiological; Hembree, 1990; Lang, 1968) are explored. The child is asked to estimate, to what extend specific statements apply to the particular situation, e.g. „I can not get a word out". Overall four situations are provided (on the eve of a math test, math homework, math class and everyday/shopping). Out of these 20 items a total score is computed with a range from 0 to 60 .

136 children at the age of 7 to 10 were included in data analyses, while the mean age was 8.33 years $(S D=0.78)$. The children attended classes from the second to the fifth grade in Potsdam and Berlin, Germany. Children were excluded if overall intelligence was found to be more than 2 standard deviations below the norm $($ T-value $<30)$. The study population involved 
more girls $(n=89)$ than boys $(n=47)$, so the gender ratio deviates significantly from equal distribution $\left(X^{2}(1)=12.79, p<.001\right)$.

The BUEGA was used to assess verbal and nonverbal intelligence as well as their performance in reading, spelling and math word problems (Esser, Wyschkon \& Ballaschk, 2007). Moreover, arithmetic performance was measured using the addition and subtraction subtests of the "Heidelberger Rechentest" (HRT, Haffner, Baro, Parzer \& Resch, 2005). A list of 40 addition/subtraction tasks is presented to the children and they are asked to solve as many problems as possible within two minutes. In addition, math anxiety (MAI), school phobia and test anxiety (PHOKI, Döpfner, Schnabel, Goletz \& Ollendick, 2006), attitudes towards mathematics and self-evaluation of mathematical competence (FRA) and trait anxiety (KAT-II; Kinder-Angst-Test; Thurner \& Tewes, 2000) have been assessed.

Results: As an index of internal consistency a coefficient, Cronbach's $\alpha$, was computed and found to be .90 . The math anxiety scale showed a mean value of $15.22(S D=11.54)$ with a range from 0 - 43.2 points. No gender differences were detected.

Further analyses showed high correlations between the math anxiety scale with trait anxiety (KAT-II) and with school phobia and test anxiety (PHOKI) $\left(r_{s}>.60, p<.001\right)$. High negative correlations were found for the relationship between MAI and FRA, indicating that the higher the levels of math anxiety, the lower the self-evaluation of mathematical performance and the lower the attitudes towards mathematics $\left(r_{s}=-.66, p<.001\right)$. The correlation coefficients showed no significant differences between boys and girls. Moreover a minor positive correlation was demonstrated between the math anxiety scale and age (in months) $\left(r_{s}=.14, p=.096\right)$.

Further results demonstrated moderate correlations between the math anxiety scale with mathematical performance (addition: $r_{s}=.395, p<.001$, subtraction: $r_{s}=.464, p<.001$ as well as math word problems: $\left.r_{s}=.419, p<.001\right)$. Partial correlations were used to control for the effect of general cognitive performance, trait anxiety, school phobia and test anxiety. These analyses indicated that the math anxiety scale was significantly and negatively correlated with mathematical performance, even after controlling for each of these variables. 
Discussion: The aim of the study was to present and psychometrically evaluate a new measure for assessing math anxiety in early primary school children. In summary, the results concerning the validity of the instrument mirror previous research results (Hembree, 1990; Devine et al., 2012; Krinzinger et al., 2007) and demonstrate a satisfying criterion validity of the MAI. The correlations between math anxiety and math performance can be classified as moderate and are therefore higher than the reported findings of other current studies with primary school children (Vukovic et al., 2013; Wu et al., 2012) or existing meta-analyses (Hembree, 1990; Ma, 1999).

It seems important to highlight that the relationship between math anxiety and math performance can be neither explained by general cognitive performance nor by trait anxiety, school phobia or test anxiety. These results are consistent with existing reports concerning math anxiety (Devine et al., 2012; Wu et al., 2012) and support the construct of math anxiety that is assessed with the MAI.

Furthermore the present study revealed no gender differences (see also Ramirez et al., in press). Literature on math anxiety demonstrates inconsistent results concerning gender differences in early primary school children. Probable reasons include the lack of standardised instruments measuring math anxiety in young children. It remains unclear whether girls experience higher levels of math anxiety or are more willing than boys to admit their anxiety (Krinzinger \& Kaufmann, 2006). It is assumed that gender differences increase with age (Dowker, 2005), and are therefore in the first grade levels not yet present. Moreover, possible self-selection effects, which were found in clinical diagnostics and treatment of learning disorders, can not be ruled out (Krinzinger \& Günther, 2013).

With respect to the sample it has to be considered that the examined children are part of a training program for enhancing numerical cognition. Therefore future studies with the aim to reveal possible sample-specific effects and to evaluate whether the results are transferable to a representative sample are needed. Standard scores should be determined subsequently.

In summary, the results of the present study suggest that a valid and reliable measure to assess math anxiety in primary school children was successfully developed. The MAI is economically to administer and was shown to be suitable for young children. Further 
investigations of the retest-reliability and the development of the relationship between math anxiety and math performance, as well as potentially later occurring gender differences are planned. For that purpose the existing children's version is aimed to be complemented by a math anxiety questionnaire for parents.

Keywords: math anxiety, primary school children, math achievement, clinical diagnostics, psychometric properties 


\section{Einleitung}

Wenn Menschen gefragt werden, woran sie sich erinnern, wenn sie an ihren früheren Mathematikunterricht zurückdenken, ist die Antwort häufig nicht nur inhaltlich geprägt, sondern auch affektiv gefärbt. Nicht selten geht dies mit aversiven oder sogar ängstlichen Emotionen einher. Eine Möglichkeit, wie diese affektive Reaktion, Angst vor Rechnen und Mathematik, bereits im Grundschulalter untersucht werden kann, steht hier im Fokus.

Richardson und Suinn (1972) definieren Mathematikangst als „...feelings of tension and anxiety that interfere with the manipulation of numbers and the solving of mathematical problems in a wide variety of ordinary life and academic situations" (S. 551). Diese Definition verdeutlicht, dass Mathematikangst sowohl in schulischen als auch in Alltagssituationen auftreten kann, in denen es zur Konfrontation mit Zahlen oder Mathematik kommt, beispielsweise im Mathematikunterricht oder beim alltäglichen Umgang mit Geld (Ashcraft, Krause \& Hopko, 2007; Richardson \& Suinn, 1972). Mathematikangst erfüllt die klassischen Merkmale einer spezifischen Phobie. So tritt Rechenangst beispielsweise stimulus- bzw. situationsgebunden bei Antizipation von oder Konfrontation mit entsprechenden Reizen auf, wird im Laufe der Lerngeschichte erworben und wirkt sich auf unterschiedlichen Angstebenen aus (Faust, 1992; Krinzinger \& Kaufmann, 2006; Saß, Wittchen, Zaudig \& Houben, 2003). Sie wird charakterisiert durch physiologische Reaktionen, negative und dysfunktionale Kognitionen und Vermeidungsverhalten (Hembree, 1990; Lang, 1968).

Neurowissenschaftliche Erkenntnisse haben zudem gezeigt, dass Mathematikangst tatsächlich schmerzt. Das heißt, bereits die Erwartung eine Rechenaufgabe lösen zu müssen oder das Rechnen selbst erzeugt bei mathematikängstlichen Erwachsenen und Kindern Aktivität in jenen Hirnregionen, welche normalerweise physische Schmerzen verarbeiten (Lyons \& Beilock, 2012; Young, Wu \& Menon, 2012). Bezüglich der unterschiedlichen Angstebenen sind insbesondere die Auswirkungen des Vermeidungsverhaltens bedeutsam. Vermeidung kann dazu führen, dass sich die Kinder weniger im Unterricht engagieren, deutlich weniger lernen und zukünftig seltener mathematikbezogene Fächer wählen (Ashcraft \& Kirk, 2001; Hembree, 1990) sowie im schlimmsten Fall auch zu Schulangst und psychosomatischen Leiden beitragen (Ashcraft, 2002; Dowker, 2005). 
In der Literatur wird ein Teufelskreis beschrieben, in dem die Mathematikangst zu negativen Erfahrungen mit Mathematik, zu Misserfolg führt, dies wiederum die Mathematikangst verstärkt und zu schlechteren Leistungen beiträgt (Devine, Fawcett, Szücs \& Dowker, 2012; Dowker, Bennett \& Smith, 2012; Krinzinger \& Kaufmann, 2006). Auch neurokonstruktivistische Modelle betonen die Bedeutung von Angst für die Entwicklung domänenspezifischer Hirnfunktionen (z. B. Kaufmann \& von Aster, 2012).

Wie häufig die Mathematikangst und andere leistungsbezogene Ängste auftreten, lässt sich bislang nicht eindeutig quantifizieren (Fehm \& Fydrich, 2011; siehe aber Pixner \& Kaufmann, 2013). So besteht beispielsweise keine Einigkeit über die Definition einer überdurchschnittlichen Mathematikangstausprägung. Einige Autoren stützen sich hierbei auf statistische Kriterien und betrachten Werte, die mindestens eine Standardabweichung über dem Stichprobenmittelwert liegen, als klinisch relevant. Bei einer derartigen Festlegung ergibt sich eine Häufigkeit von 16 bis 20\% (Ashcraft et al., 2007; Ashcraft \& Ridley, 2005). Die Ergebnisse der PISA-Studie 2003 weisen darauf hin, dass in der Gruppe 15-jähriger Schüler und Schülerinnen international ca. 30\% von Symptomen der Mathematikangst betroffen sind (OECD, 2004, Tabelle 3.8).

Mathematikangst ist nach Hopko, McNeil, Zvolensky und Eifert (2001) eine leistungsbezogene Angststörung und weist zahlreiche Überschneidungsbereiche mit verschiedenen anderen Angstformen auf, wobei sich die deutlichsten Zusammenhänge zur Prüfungsangst ergeben (Devine et al., 2012; Hembree, 1990). So lag beispielsweise die Korrelation zwischen Mathematikangst und Prüfungsangst für Jungen bei $r=.76$, während sie für Mädchen $r=.36$ betrug (Devine et al., 2012). Mathematikangst geht weiterhin mit negativen Einstellungen zu Mathematik, einer geringen Freude an Mathematik und einem niedrigen Selbstbewusstsein in diesem Fach einher (Ashcraft \& Kirk, 2001; Hembree, 1990; Krinzinger et al., 2007). Insgesamt ist Mathematikangst trotz der zahlreichen Überschneidungen mit anderen Ängsten und verwandten Variablen dennoch als eigenständiges Konstrukt zu betrachten (Dew, Galassi \& Galassi, 1984; Hembree, 1990; Punaro \& Reeve, 2012). In diesem Sinne stellten auch Dreger und Aiken (1957) fest, dass Mathematikangst deutlich von der allgemeinen Ängstlichkeit abzugrenzen ist und auch in Abwesenheit einer erhöhten Ängstlichkeitsausprägung auftreten kann (Rubinsten \& Tannock, 2010). Zudem scheint 
Mathematikangst lediglich schwach mit allgemeiner Intelligenz assoziiert zu sein (Ashcraft, 2002; Maloney \& Beilock, 2012).

Besondere Bedeutung kommt der Untersuchung des Zusammenhangs zwischen Mathematikangst und Rechenleistung zu. In zahlreichen Studien ließen sich negative Beziehungen zur Mathematikleistung, vor allem für die Sekundarstufe, nachweisen. So fanden sich Korrelationen zwischen der Mathematikleistung und der Mathematikangst von $r=-.27$ bis $r=-.34$ (Hembree, 1990; Ma, 1999). Zur Erklärung der Wirkungsmechanismen wird der Einfluss der Mathematikangst auf das Arbeitsgedächtnis diskutiert (z. B. Ashcraft \& Kirk, 2001). Matheangst kann wie eine parallele Aufgabenstellung betrachtet werden, die kognitive Ressourcen bei der Lösung der Mathematikaufgaben beansprucht (Ashcraft \& Krause, 2007; Ashcraft \& Kirk, 2001; Ashcraft \& Moore, 2009). Dennoch ist die Richtung des Zusammenhangs bislang unklar, wobei eher von einer bidirektionalen Wechselwirkungsbeziehung auszugehen ist (Krinzinger, Kaufmann \& Willmes, 2009).

Die Mehrzahl der Befunde zur Mathematikangst stützen sich auf Studien, die sich auf junge Erwachsene (z. B. Ashcraft \& Krause, 2007; Hopko, Mahadevan, Bare \& Hunt, 2003; Maloney, Ansari \& Fugelsang, 2011) bzw. Kinder der Mittelstufe (Imbo \& Vandierendonck, 2007; Jain \& Dowson, 2009; Meece, Wigfield \& Eccles, 1990) beziehen. Erst in den letzten Jahren wird der Fokus stärker auf das frühe Grundschulalter gelegt (Krinzinger et al., 2009; Vukovic, Kieffer, Bailey \& Harari, 2013; Wood et al., 2012; Wu, Barth, Amin, Malcarne \& Menon, 2012). Dazu wurden im englischen (Ramirez, Gunderson, Levine \& Beilock, in Druck; Vukovic et al., 2009; $\mathrm{Wu}$ et al., 2012) sowie im deutschen Sprachraum (Krinzinger et al., 2009) altersgerechte Instrumente zur Erfassung der Mathematikangst entwickelt. Als Grundlage dienten beispielsweise die Math Anxiety Rating Scale - Elementary (MARS-E, Suinn, Taylor \& Edwards, 1988) sowie der Math Anxiety Questionnaire (MAQ, Thomas \& Dowker, 2000).

Mit diesen Instrumenten ließen sich Unterschiede zwischen den Kindern und Zusammenhänge zwischen der Mathematikangst und der Mathematikleistung bereits in der zweiten bzw. dritten Klasse nachweisen (Punaro \& Reeve, 2012; Ramirez et al., in Druck; Vukovic et al., 2009; Wu et al., 2012). Des Weiteren weisen die Befunde auf Unterschiede zwischen verschiedenen Typen von Rechenaufgaben und auf Wechselwirkungen mit Arbeitsgedächtnisleistungen hin. Beispielsweise ließ sich mittels der Scale for Early Mathematics Anxiety (SEMA) ein negativer Zusammenhang zum mathematischen 
Schlussfolgern, jedoch nicht zur Lösung von Rechenoperationen finden (Wu et al., 2012). Darüber hinaus fanden Ramirez et al. (in Druck) zwar einen Zusammenhang zwischen Mathematikangst und Mathematikleistung, jedoch nur für solche Kinder, die eine hohe Ausprägung von Arbeitsgedächtnis-Kapazitäten aufwiesen.

Das einzige deutschsprachige Verfahren für das frühe Grundschulalter (1. - 3. Klasse) entwickelten Krinzinger und Kollegen (2007). Der Fragebogen für Rechenangst (FRA) für 6bis 9-jährige Kinder basiert auf dem MAQ (Thomas \& Dowker, 2000) und erfasst die Selbsteinschätzung der Rechenleistung, die Einstellung zum Rechnen sowie die Gefühle von Traurigkeit und Sorge bei schlechter Rechenleistung. Während sich für die Selbsteinschätzung und Einstellung zum Rechnen die erwarteten Zusammenhänge zur Rechenleistung nachweisen ließen, fanden sich nur geringe Zusammenhänge zwischen der Rechenleistung und den rechenangstbezogenen Skalen (Krinzinger et al., 2007). Im Langzeitverlauf ließ sich ebenfalls kein Einfluss der Rechenangst auf die spätere Rechenleistung und umgekehrt nachweisen (Krinzinger et al., 2009). Die Autoren vermuten, dass eventuell die Formulierung der Fragen für junge Kinder schwer verständlich war. Sie empfehlen eine direktere Erfassung der Angst anstelle von Fragen nach Sorge und Traurigkeit bei schlechter Rechenleistung (Krinzinger et al., 2009).

\subsection{Fragestellungen}

Aus der Literaturübersicht lässt sich ableiten, dass im deutschen Sprachraum bisher kein valides Instrument zur Erfassung der Mathematikangst im frühen Grundschulalter vorliegt (z. B. Krinzinger et al., 2009). Um diese Lücke zu schließen, ist das Ziel dieser Studie ein kindgerechtes, ökonomisches Verfahren zu entwickeln und dies auf seine psychometrischen Eigenschaften $\mathrm{zu}$ untersuchen. Der Fokus liegt dabei auf den Fragen: Wie reliabel ist das Instrument und wie gut ist seine kriterienbezogene Validität? Dabei wird trotz der uneinheitlichen Studienlage erwartet, dass sich ein bedeutsamer negativer Zusammenhang zwischen der Mathematikangst und der Rechenleistung nachweisen lässt.

\subsubsection{Das Mathematikangstinterview (MAI)}

Das Mathematikangstinterview kombiniert zwei Fragestellungen. Dazu wird eine rechenspezifische Situation sprachlich und bildlich präsentiert und das Kind gebeten, sich diese genau vorzustellen. Erfragt wird zunächst die Angstintensität, wobei das Kind mittels eines 
Angstthermometers von 0 bis 10 selbst einstellen kann, wie viel Angst es in der jeweiligen Situation hat. Auf diese Weise wird die Anregung von Krinzinger und Kollegen (2009) aufgenommen, die Kinder direkt nach der Angst zu fragen.

Im zweiten Schritt wird auf die unterschiedlichen Ebenen der Angst (affektiv, kognitiv, verhaltensbezogen und physiologisch, Hembree, 1990; Lang, 1968) eingegangen. Dabei wird das Kind gebeten einzuschätzen, wie sehr die Aussagen in den jeweiligen Situationen zutreffen, z. B. „Ich bin nervös“ oder „Ich bekomme kein Wort heraus“. Dabei kann es auf Visualisierungen der vier Antwortalternativen zeigen (,stimmt gar nicht“, „stimmt ein bisschen“, „stimmt ziemlich“ und „stimmt ganz genau“, 0 - 3 Punkte). Es werden vier verschiedene Situationen vorgegeben (Vorabend der Mathematikarbeit, Mathehausaufgaben, Unterricht und Alltag/Einkaufen), wobei das Bildmaterial geschlechtsspezifisch ist. Insgesamt besteht das Interview aus vier Items zur Angstintensität und 16 Items zur Auswirkung auf den Angstebenen. Zur Auswertung wird ein Gesamtwert über die Summe aller Items bestimmt (Range 0-60), wobei höhere Werte mit höherer Angst einhergehen. Um auszuschließen, dass die Items zur Angstintensität höheren Einfluss auf den Gesamtwert haben, werden diese zuvor auf die Skalierung von 0-3 überführt, indem sie durch 3.33 dividiert werden.

\section{Methodik}

Die Rekrutierung der Kinder erfolgte in Berlin und Potsdam im Rahmen eines BMBFgeförderten Projektes zur Evaluation eines computerbasierten Rechentrainings. Die Testungen erfolgten im Einzelsetting durch Diplompsychologen bzw. intensiv geschulte Studentinnen und umfassten ca. 90-120 Minuten. Im Rahmen der Erhebung wurden zunächst die kognitiven Leistungen der Kinder untersucht und im Anschluss die Selbsteinschätzungen erfragt.

\subsection{Instrumente}

\subsubsection{Basisdiagnostik Umschriebener Entwicklungsstörungen im Grundschulalter (BUEGA)}

Die BUEGA (Esser, Wyschkon \& Ballaschk, 2007) dient der Erfassung der verbalen und nonverbalen Intelligenz, der Lese-, Rechtschreib- und Rechenleistung. Die Untertests zur Erfassung des sprachlichen sowie des nicht-sprachlichen schlussfolgernden Denkens werden zu einem Gesamtwert „Gesamtintelligenz“ zusammengefasst. Die Leseleistung wird sowohl über die Erhebung der Lesezeit als auch der Lesegenauigkeit ermittelt. Die Rechtschreibleistung wird 
über das korrekte Schreiben von Worten (genauer von Graphemen) erfasst. Der Untertest Rechnen überprüft die Leistung in den vier Grundrechenarten, indem das Kind eine Reihe von Textaufgaben im Kopf lösen soll. Die internen Konsistenzkoeffizienten sind für die einzelnen Klassenstufen als ausreichend bis hoch einzuschätzen $(\alpha=.81$ bis $\alpha=.95)$.

\subsubsection{Heidelberger Rechentest (HRT 1-4)}

Anhand des HRT 1-4 (Haffner, Baro, Parzer \& Resch, 2005) werden die Rechenoperationen Addition und Subtraktion erfasst. Dabei wird insbesondere die Rechenflüssigkeit überprüft, indem den Kindern eine Reihe von einstelligen bzw. zweistelligen Aufgaben schriftlich präsentiert wird, von denen sie innerhalb von zwei Minuten so viele wie möglich korrekt lösen sollen. Die Reliabilität wurde über die Retest-Reliabilität nach ein bis zwei Wochen ermittelt und ist mit $r_{t t}=.82$ für die Addition und $r_{t t}=.86$ für die Subtraktion als hoch zu bewerten.

\subsubsection{Kinder-Angst-Test (KAT-II)}

Zur Erfassung der allgemeinen Ängstlichkeit, der Trait-Angst, diente die Form A des KATII (Thurner \& Tewes, 2000). Erfragt wird die Zustimmung zu 20 Items, die sich beispielsweise auf Sorgen vor unterschiedlichen Ereignissen beziehen. Die Autoren berichten eine zufriedenstellende interne Konsistenz der Skala von $\alpha=.81$ (Thurner \& Tewes, 2000). Die Retest-Reliabilität ermittelt nach einem 2-Wochen-Intervall liegt bei $r_{t t}=.80$.

\subsubsection{Phobiefragebogen für Kinder und Jugendliche (PHOKI)}

Zur Erfassung der schulbezogenen Angst diente die Skala „Schul- und Leistungsängste“ des PHOKI (Döpfner, Schnabel, Goletz \& Ollendick, 2006). Die Kinder geben an, wie häufig (gar nicht, manchmal, oft) sie vor den genannten Situationen Angst haben. Dazu zählen Situationen, wie beispielsweise „,vor der Klasse etwas sagen zu müssen“. Die Reliabilitätsschätzungen wurden über die interne Konsistenz nach Cronbachs Alpha ermittelt und sind als zufriedenstellend zu bewerten $(\alpha=.78)$.

\subsubsection{Fragebogen für Rechenangst (FRA)}

Aus dem Fragebogen für Rechenangst für 6- bis 9-jährige Kinder (Krinzinger et al., 2007) wurden die Skala „Selbsteinschätzung der Rechenleistung“, z. B. „Wie gut bist Du beim Rechnen?“, und die Skala „Einstellung zum Rechnen“, z. B. „Wie gerne rechnest Du?“, verwendet. Aufgrund der Ergebnisse zur Konstruktvalidität empfehlen die Autoren, beide 
Skalen zur kombinierten Skala „Selbsteinschätzung und Einstellung“ zusammenzufassen (Krinzinger et al., 2007). Die interne Konsistenz, ermittelt über Cronbachs Alpha, ist als zufriedenstellend zu bewerten ( $\alpha=.84$ für Zweitklässler [2. Halbjahr] und $\alpha=.85$ für Drittklässler).

\subsection{Stichprobe}

Es konnten 136 Kinder im Alter von 7 bis 10 Jahren in die Datenanalyse eingeschlossen werden, wobei das mittlere Alter bei 8.33 Jahren $(S D=0.78)$ lag. Die Kinder besuchten die zweite bis fünfte Klassenstufe. Es wurden alle Kinder ausgeschlossen, deren Gesamtintelligenz mehr als zwei Standardabweichungen unter dem Mittelwert der Altersgruppe lag (d. h. T-Wert < 30). Das Geschlechterverhältnis (89 Mädchen zu 47 Jungen) weicht signifikant von einer Gleichverteilung ab $\left(X^{2}(1)=12.97, p<.001\right)$. In Tabelle 1 sind die mittleren Intelligenz-, Lese-

, Rechtschreib- und Rechenleistungen sowie die mittleren Werte zur Selbsteinschätzung der Kinder, getrennt nach Geschlecht, dargestellt. Es wird deutlich, dass sich keine Geschlechtsunterschiede für die Rechen- und Leseleistungen sowie die Angstmaße zeigen. Jedoch ergeben sich Leistungsunterschiede für die Rechtschreibung und die Gesamtintelligenz dahin gehend, dass Jungen geringere Werte aufweisen.

>> Tabelle 1 hier einfügen $\ll<<$

\subsection{Statistische Verfahren}

Zur Überprüfung der Zusammenhangshypothesen kommen Korrelationsanalysen nach Pearson bzw. nach Spearman zum Einsatz, wenn die Variablen nicht normalverteilt sind. Dabei gelten Korrelationskoeffizienten von $r \geq .40$ als moderat und von $r \geq .60$ als hoch (Field, 2009).

Zur Bewertung des Unterschieds zwischen den Korrelationen wird die Effektgröße $q$ berechnet (Cohen, 1988). Nach Cohen (1988) sind Werte von $q=.10-.29$ als gering, Werte von $q=.30-.49$ als mittel und Werte von $q \geq .50$ als groß einzuschätzen. Für den Vergleich zweier Korrelationskoeffizienten wird auf die Berechnung von $z$-Werten zurückgegriffen. 


\section{Ergebnisse}

\section{Deskriptive Statistik zum Mathematikangstinterview (MAI)}

Die Gesamtskala weist einen Mittelwert von $15.22(S D=11.54)$ auf bei einem Range von 0 bis 43.2 Punkten. Die Skala ist rechtsschief verteilt (Schiefe $=0.59, S D=0.21)$, d. h., dass viele Kinder niedrige Werte und wenige Kinder hohe Werte angeben. Es zeigt sich kein signifikanter Geschlechtsunterschied $(z$-Wert $=0.881, p>.1)$. Während Mädchen einen Mittelwert der Angstintensität von $15.59(S D=10.92)$ aufweisen, liegt dieser bei Jungen bei $14.53(S D=$ 12.73).

Die Reliabilität der Skala wird über die interne Konsistenz nach Cronbachs Alpha ermittelt. Diese liegt bei $\alpha=.90$, wobei die Trennschärfen der Items der Skala von $r_{\text {it }}=.25$ bis $r_{\text {it }}$ $=.72$ reichen.

\section{Validität}

Es bestehen hohe positive Korrelation zwischen dem MAI und der allgemeinen Ängstlichkeit (KAT-II) sowie der Schul- und Leistungsangst (PHOKI). Zudem ergeben sich hohe negative Korrelationskoeffizienten für den Zusammenhang zwischen dem MAI und dem FRA. Je höher die Mathematikangstwerte, desto geringer die Selbsteinschätzung der eigenen Rechenleistungen und desto negativer wird das Rechnen bewertet und umgekehrt. In Tabelle 2 sind die Korrelationskoeffizienten für das MAI sowie die Interkorrelationen zwischen den Verfahren dargestellt, wobei alle Ergebnisse als statistisch bedeutsam zu bewerten sind.

>> Tabelle 2 hier einfügen $\ll<<$

In Tabelle 3 sind die Korrelationskoeffizienten für die Stichprobe der Jungen und der Mädchen separat dargestellt. Es fällt auf, dass sich die Korrelationskoeffizienten kaum unterscheiden. Die Effektgrößen für die Unterschiede liegen unter .30 und sind daher als schwach zu bewerten.

>> Tabelle 3 hier einfügen $\ll<<$

Darüber hinaus ergibt sich für die untersuchte Altersgruppe eine sehr geringe positive Korrelation zwischen der Mathematikangst und dem Alter (in Monaten) der Kinder $\left(r_{s}=.143\right)$, die marginal signifikant ist $(p=.096)$. Demnach geht ein höheres Alter mit höheren Mathematikangstwerten einher. 
Zur Überprüfung der Hypothese eines negativen Zusammenhangs von Rechenleistungen und Mathematikangst werden ebenso Korrelationsanalysen nach Spearman bestimmt (siehe Tabelle 2 und Tabelle 3). Dabei zeigen sich überwiegend moderate Korrelationen zwischen der Mathematikangst und der Addition HRT, Subtraktion HRT sowie dem Rechentest der BUEGA. Erwartungsgemäß gehen geringere Rechenleistungen mit höheren Angstwerten einher und umgekehrt.

Zur Bewertung des Einflusses der allgemeinen kognitiven Leistung wurden die genannten Zusammenhänge unter Kontrolle der Gesamtintelligenz überprüft.

Die Korrelationskoeffizienten für den Zusammenhang zwischen der Gesamtintelligenz und der Mathematikangst $\left(r_{s}=-.207, p<.05\right)$ ist als gering zu bewerten. Wird die Gesamtintelligenz (BUEGA) als Kontrollvariable in die Partialkorrelation einbezogen, verändern sich die zuvor beschriebenen Ergebnisse kaum (siehe Tabelle 4). Es bestehen weiterhin geringe bis moderate negative signifikante Korrelationen zwischen der Mathematikangst und der Rechenleistung.

Zur Überprüfung der Spezifität des Zusammenhangs wurden die Korrelationskoeffizienten zwischen der Angst und der Lese- bzw. Rechtschreibleistung unter Kontrolle der Gesamtintelligenz bestimmt. Der Partialkorrelationskoeffizient für die Leseleistung ist sehr gering und nicht signifikant. Für die Rechtschreibleistung zeigt sich eine geringe negative signifikante Korrelation zur Mathematikangst. In Tabelle 4 sind ebenso die Partialkoeffizienten der Jungen- und Mädchenstichprobe angegeben. Wiederum zeigen sich vergleichbare Ergebnisse, mit einer Ausnahme. Während für Jungen der Zusammenhang zwischen der Mathematikangst und der Rechtschreibleistung praktisch und statistisch unbedeutsam ( $r=.036$, $p>.10)$ ist, zeigt sich ein signifikanter Zusammenhang für die Gruppe der Mädchen $(r=-.284$, $p<.05)$, welcher signifikant höher als in der Gruppe der Jungen ist.

>> Tabelle 4 hier einfügen $\ll<$

Zusätzlich wird überprüft, ob sich der Zusammenhang zwischen der Mathematikleistung und der Mathematikangst auf die allgemeine Ängstlichkeit oder die Schul- und Leistungsangst zurückführen lässt. Dazu kommen ebenfalls Partialkorrelationen zum Einsatz. Die Ergebnisse sind in Tabelle 4 dargestellt. Die Zusammenhänge zwischen der Mathematikangst und der Rechenleistung bleiben für beide Kontrollvariablen (allgemeine Ängstlichkeit; Schul- und 
Leistungsangst) stabil und sind weiterhin als gering bis moderat einzuschätzen. Es bestehen keine bedeutsamen Unterschiede zwischen den Geschlechtern.

\section{Diskussion}

Das Ziel dieser Studie war die Entwicklung und psychometrische Überprüfung eines Verfahrens zur Erfassung der Mathematikangst im frühen Grundschulalter. Dazu wurde das neu entwickelte Mathematikangstinterview (MAI) an einer Stichprobe von 7- bis 10-jährigen Kindern erprobt. Die ersten Ergebnisse verdeutlichen, dass das Interview eine hohe Reliabilität, ermittelt über die interne Konsistenz, aufweist.

Insgesamt spiegeln die Ergebnisse zur Validität bisherige Untersuchungsergebnisse zur Mathematikangst wider und legen eine zufriedenstellende Kriteriumsvalidität des MAI nahe.

Die beobachteten Zusammenhänge zwischen der Mathematikangst und der Schul- und Leistungsangst sowie zur allgemeinen Ängstlichkeit sind als hoch zu bewerten und liegen etwas über den Werten bisheriger Untersuchungen (Devine et al., 2012; Hembree, 1990). Des Weiteren legen die Ergebnisse einen hohen Zusammenhang zwischen der Mathematikangst und der Selbsteinschätzung der Rechenleistung sowie der persönlichen Einstellung zur Mathematik nahe. Dies bestätigt frühere Befunde für das Jugend- und Erwachsenenalter (Chipman, Krantz \& Silver, 1992; Ma, 1999) sowie für das Grundschulalter (Krinzinger et al., 2007).

Die Zusammenhänge zwischen der Mathematikangst und den Rechenleistungen liegen im geringen bis mittleren Bereich und sind etwas höher als in den aktuellen Arbeiten für Grundschulkinder (Vukovic et al., 2013; Wu et al., 2012) sowie den verfügbaren Meta-Analysen (Hembree, 1990, Ma, 1999). Zudem ergeben sich für die Lösung von einfachen arithmetischen Aufgaben (Addition und Subtraktion) ebenso moderate Korrelationen zum Mathematikangstinterview. Dieses Ergebnis ließ sich in vorhergehenden Arbeiten (Wu et al., 2012) nicht zeigen. Möglicherweise könnte die Erfassung der Rechenleistung als Speed-Test aufgrund des bestehenden Zeitdrucks dazu beitragen, dass die Zusammenhänge höher sind. Es ist $\mathrm{zu}$ vermuten, dass die Mathematikangst, insbesondere angstbezogene Gedanken, die Arbeitsgeschwindigkeit beeinträchtigt haben könnten (Hopko, Ashcraft, Gute, Ruggiero \& Lewis, 1998).

Hervorzuheben ist der Befund, dass der Zusammenhang zwischen Mathematikangst und Rechenleistung weder durch die allgemeine kognitive Leistung noch durch die allgemeine 
Ängstlichkeit oder Schul- und Leistungsangst zu erklären ist. Dieses Resultat entspricht bestehenden Befunden zur Mathematikangst (Devine et al., 2012; Wu et al., 2012) und stützt das Konstrukt der Mathematikangst, das durch das MAI erfasst wird. Zudem ließen sich keine bedeutsame Korrelation zum Lesen und nur eine geringe zur Rechtschreibung aufdecken, was auf die Spezifität des Verfahrens hinweist. Lediglich für Mädchen ergab sich eine geringe signifikante Korrelation zwischen dem MAI und der Rechtschreibung. Inwiefern es sich dabei um ein zufälliges Ergebnis oder einen replizierbaren und demnach erklärungsbedürftigen Befund handelt, sollte im Rahmen weiterer Erhebungen überprüft werden.

Es zeigt sich eine praktisch unbedeutende positive Korrelation der Mathematikangst mit dem Alter der Kinder $\left(r_{s}=.143\right)$. Dies entspricht früheren Untersuchungen (Krinzinger et al., 2007; Ramirez et al., in Druck), die ebenfalls keine systematischen Veränderungen der Rechenangstausprägung und -häufigkeit im frühen Grundschulalter fanden. Dagegen ist für die Sekundarstufe ein Anstieg der Mathematikangst mit zunehmendem Alter anzunehmen, wobei der Gipfel in der neunten und zehnten Klasse anzusiedeln ist (Hembree, 1990; Baloğlu \& Koçak, 2006).

Zudem ließen sich in der vorliegenden Studie keine Geschlechtsunterschiede aufdecken. Dies steht im Kontrast zu Untersuchungen im Jugend- und Erwachsenenalter (Hembree, 1990). Im Grundschulalter finden sich uneinheitliche Befunde. Während einige Studien eine höhere Angstintensität bei Mädchen finden (Ashcraft \& Faust, 1994; Hyde, Fennema \& Lamon, 1990; Yüksel-Şahin, 2008), berichten andere Studien keine Geschlechtsunterschiede (Chinn, 2009; Dowker et al., 2012; Ma \& Xu, 2004; Newstead, 1998; Punaro \& Reeve, 2012; Ramirez et al., in Druck). Diese Inkonsistenz der Befunde ist eventuell auf eine fehlende Standardisierung und einheitliche Nutzung von Verfahren zur Erhebung der Mathematikangst bei jüngeren Kindern zurückzuführen. Weiterhin ist nicht vollständig geklärt, ob Mädchen tatsächlich höhere Angstintensitäten erleben oder diese lediglich eher berichten (Krinzinger \& Kaufmann, 2006). Insgesamt liegt noch sehr wenig Forschung zu Geschlechtsunterschieden im Grundschulalter vor. Es wird angenommen, dass Geschlechtsunterschiede mit dem Alter zunehmen (Dowker, 2005) und daher in den ersten Klassenstufen noch weniger deutlich sind. In Bezug auf die Stichprobe ist zudem zu berücksichtigen, dass ein Zuweisungs- bzw. Selektionsbias denkbar ist, wie er für die klinische Praxis häufiger berichtet wird (Krinzinger \& Günther, 2013). Eventuell wurden insbesondere Jungen mit Rechenschwächen und zusätzlichen Leistungsschwächen und 
psychischen Symptomen zur Förderung vorgestellt. Darauf könnten die geringeren Rechtschreibleistungen der Jungen verglichen mit der Gruppe der Mädchen hinweisen. Insgesamt ist der Anteil der Jungen in der Gruppe mit Rechenschwächen (32\%) mit jenem in der Gruppe mit durchschnittlichen Rechenleistungen (36 \%) jedoch vergleichbar.

In der vorliegenden Studie ließ sich querschnittlich zeigen, dass eine hohe Mathematikangst mit geringen Rechenleistungen einhergeht. Ashcraft (2002) nimmt an, dass die eigentliche Mathematikleistung der mathematikängstlichen Kinder durch die Mathematikangst herabgesetzt wird und die tatsächlichen mathematischen Kompetenzen nach der Behandlung sichtbar werden. Dies unterstreichen die Ergebnisse des Reviews von 122 Studien für Jugendlichen mit Rechenschwierigkeiten (Hembree, 1990), wobei eine effektive Behandlung der Mathematikangst zu einer Verbesserung der Mathematikleistung führte. Diese Befunde haben eine weitreichende Bedeutung und betonen die affektive Komponente bei der Erfassung der Mathematikleistung. Darüber hinaus zeigte eine aktuelle deutschsprachige Untersuchung (Lambert \& Spinath, 2013) den bedeutsamen Effekt einer kognitiven (dyskalkuliespezifischen) Intervention (Wasserglasmethode) auf das schulbezogene psychische Funktionsniveau. Rechenschwache Kinder zeigten neben einer verbesserten Rechenleistung eine verminderte psychische Belastung (z. B. Prüfungsangst, Schulunlust), wobei methodische Schwierigkeiten der Untersuchung (z. B. kleine Stichprobenumfänge, mögliche Gruppenselektionseffekte, keine unbehandelte Kontrollgruppe bzw. vollständig kontrollierte Alternativbedingung) beachtet werden sollten. Der Effekt der verringerten psychischen Belastung wurde durch die Verbesserung der Mathematiknote mediiert. $\mathrm{Zu}$ den Wirkmechanismen, die zu einer verringerten Symptombelastung, unter anderem verminderten Ängstlichkeit, führen, lassen sich bislang keine Aussagen treffen. Diskutiert werden könnten die verbesserte Mathematikleistung und die damit verbundenen häufigeren Erfolgserlebnisse, die vermehrte Beschäftigung mit dem angstauslösenden Objekt oder auch der Einfluss der Bewertung durch den Lehrer.

In zukünftigen Arbeiten soll zudem der Fokus noch stärker auf interindividuelle Unterschiede zwischen Kindern mit und ohne Dyskalkulie gelegt werden. Die Ergebnisse der wenigen Studien (Rubinsten \& Tannock, 2010) weisen auf höhere Mathematikangstwerte bei Kindern mit Dyskalkulie hin. Zudem stehen Längsschnittstudien mit validen Erhebungsverfahren zur Bewertung eines Kausalzusammenhangs zwischen Mathematikangst und Leistung noch aus. 


\subsection{Limitationen}

In dieser Studie wurde das neu entwickelte MAI auf seine psychometrischen Eigenschaften überprüft. Neben der internen Konsistenz ist die Bewertung der Retest-Reliabilität wünschenswert. Diese ist bislang noch nicht für ein angemessenes Zeitintervall untersucht worden. Zudem stehen Untersuchungen $\mathrm{zu}$ den verschiedenen Angst-Ebenen und deren Zusammenhängen mit den Angstmaßen sowie Rechenleistungen aus. Darüber hinaus ist darauf hinzuweisen, dass die verwendeten Fragebögen zur Erfassung der allgemeinen Ängstlichkeit (KAT-II) und der Schul- und Leistungsängste (PHOKI) eigentlich erst für Kinder ab acht bzw. neun Jahren eingesetzt werden sollten. Um eventuelle Leseverständnisprobleme zu vermeiden, wurden die Fragen im Einzelsetting vorgelesen. In Bezug auf die untersuchte Stichprobe gilt zu berücksichtigen, dass es sich um Kinder handelt, die an einer Rechentrainingsstudie teilnahmen. Diese umfasst Kinder mit Rechenschwierigkeiten $(n=47)$ sowie Kinder mit mindestens durchschnittlichen Rechenleistungen, die gern rechnen und ihre Rechenleistungen noch verbessern wollten $(n=89)$. Zur Differenzierung der Gruppen diente der Mittelwert der Rechenleistungen (HRT Addition, Subtraktion und BUEGA Rechnen), wobei als Kriterium ein T-Wert von 40 (1 SD unter dem Mittelwert der Klassenstufe) herangezogen wurde. Um stichprobenspezifische Effekte $\mathrm{zu}$ erfassen, ist eine Untersuchung der Übertragbarkeit der Ergebnisse auf eine repräsentative Stichprobe wünschenswert. Erst dann sollte eine Berechnung von Normwerten vorgenommen werden.

\subsection{Schlussfolgerung}

Zusammenfassend weisen die Untersuchungsergebnisse darauf hin, dass es gelungen ist, ein kindgerechtes und ökonomisches Verfahren zur reliablen und validen Erfassung der Mathematikangst im frühen Grundschulalter $\mathrm{zu}$ entwickeln. Nach einer weiterführenden Überprüfung der Retest-Reliabilität des Mathematikangstinterviews sind Untersuchungen insbesondere zur Entwicklung des Zusammenhangs von Mathematikangst und Rechenleistungen sowie $\mathrm{zu}$ eventuell später auftretenden Geschlechtsunterschieden geplant. Dazu soll die bisherige Kinderversion um einen Mathematikangstfragebogen für Eltern erweitert werden. 


\section{Danksagung}

Wir bedanken wir uns bei allen Kindern sowie deren Eltern für ihre Teilnahme an dieser Studie.

Für die finanzielle Unterstützung danken wir dem Bundesministerium für Bildung und Forschung (BMBF, 01GJ1011). Darüber hinaus bedanken wir uns bei den studentischen Mitarbeiterinnen, die uns während der Datenerhebung und -eingabe intensiv unterstützt haben: Nadine Häusler, Sarah Müller und Alexandra Schmitterer. 


\section{Infoboxen}

\subsection{Implikationen für die Praxis}

Im Prozess der Diagnostik von Rechenstörungen sollte neben der ausführlichen Erfassung domänenspezifischer und domänenübergreifender kognitiver Leistungen auch das sozioemotionale Befinden berücksichtigt werden (Kaufmann \& von Aster, 2012). Dies schließt Verfahren zur Erfassung des Selbstwertgefühls, von depressiven Symptomen sowie von Schulund Leistungsängsten ein. Zur Erhebung der auf Mathematik bezogenen Ängste stellt das in dieser Studie entwickelte und überprüfte Mathematikangstinterview ein kindgerechtes und ökonomisches Instrument dar.

Durch den Einsatz des Verfahrens ließ sich zeigen, dass bereits in den ersten Klassenstufen Unterschiede zwischen den Kindern hinsichtlich der Ausprägung der Mathematikangst bestehen. Da sich für die höheren Schulklassen negative Auswirkungen der Mathematikangst auf die weitere persönliche und berufliche Entwicklung zeigen ließen (Hembree, 1990), ist es zunächst wichtig, die Übertragbarkeit auf das frühe Grundschulalter zu überprüfen. Sollten sich die Befunde bestätigen, bietet die frühe Erfassung der fachspezifischen Ängste die Möglichkeit zur Entwicklung und Implementierung von frühzeitigen Interventionen (Ramirez et al., in Druck) und damit zu einer Verbesserung des Rechnenlernens.

Auf die Bedeutung der Behandlung der Mathematikangst wies Hembree (1990) in seinem Review von 122 Studien für Jugendlichen mit Rechenschwierigkeiten hin. So trugen effektive Behandlungen der Mathematikangst deutlich zu einer Verbesserung der Mathematikleistung bei. Kamann und Wong (2002) konnten dies auch für jüngere Kinder zeigen. Sie führten eine angstgerichtete Intervention bei Kindern mit Rechenstörungen durch und konnten zeigen, dass die verbesserten Coping-Strategien $\mathrm{zu}$ einer verminderten Mathematikangst und einer verbesserten Rechenleistung führten, ohne dass die Rechenfertigkeiten zusätzlich trainiert wurden.

Eine Verbesserung der Angstsymptomatik ist insbesondere dann zu erwarten, wenn erfolgreiches, auf den angstbesetzten Gegenstand gerichtetes, Bewältigungslernen stattfinden kann (vgl. Lambert \& Spinath, 2013). Erste Resultate einer eigenen Trainingsstudie zeigen, dass 
die durch tägliches Üben erzielten Leistungsfortschritte mit einer Verminderung der Mathematikangst einhergehen.

Da sich die Mathematikleistung und die Mathematikangst vermutlich gegenseitig beeinflussen (Krinzinger et al., 2009), unterstreichen die Befunde den Bedarf frühzeitiger Diagnostik, um langfristig den Teufelskreis zwischen Mathematikangst, Vermeidung von Mathematik und schlechten Mathematikleistungen $\mathrm{zu}$ durchbrechen (Devine et al., 2012; Krinzinger \& Kaufmann, 2006).

\subsection{Forschungsmethoden}

Im Rahmen dieser Studie wurde ein Verfahren zur Erfassung der Mathematikangst entwickelt. Aufgrund der zu untersuchenden Altersgruppe ( 7 - 10 Jahre) wurde als Methode ein standardisiertes Interview gewählt. Dadurch werden eventuelle Probleme der Leseleistung sowie des Leseverständnisses vermieden und die Durchführung kann kindgerechter gestaltet werden.

Beim Mathematikangstinterview wird für die Vorgabe von Situationen auf geschlechtsspezifisches Bildmaterial zurückgegriffen. Dies soll es den Kindern erleichtern, sich die Situationen vorzustellen, wobei auf emotionale Gesichtsausdrücke, die eine affektive Reaktion nahe legen, verzichtet wurde. Zudem müssen die Antworten nicht verbal erfolgen. Beispielsweise bewerten die Kinder ihre Angst, indem sie diese über ein Angstthermometer von „gar keine Angst“ bis „ganz viel Angst“ einschätzen.

Zur Ermittlung der psychometrischen Eigenschaften des Mathematikangstinterviews kommen Reliabilitäts- und Validitätsanalysen zum Einsatz. Die Reliabilität gibt den Grad der Genauigkeit eines Messinstrumentes an, während die Validität angibt, ob ein Test tatsächlich das misst, was er zu messen vorgibt (vgl. Bühner, 2006, S. 60f.). Die Validitätsbewertung erfolgte über Rangkorrelationsanalysen nach Spearman, da die Mathematikangstvariable nicht normal verteilt war. Insbesondere bei psychischen Auffälligkeiten sind die Variablen überwiegend rechtsschief verteilt, da mehr Kinder angeben, geringe Probleme zu haben, während nur wenige Kinder über hohe Problemausprägungen berichten (Bühner, 2006). Mittels Partialkorrelationen wurde der Zusammenhang zwischen den Skalen des MAI und der Rechenleistungen geprüft, indem der Einfluss einer dritten Variable z. B. der Gesamtintelligenz rechnerisch „,ausgeschaltet“ wurde (Bortz, 1999). Zudem wurde die Höhe der Korrelationskoeffizienten über das Effektstärkemaß $q$ sowie die Bestimmung eines $z$-Wertes 
verglichen. Zur Berechnung der Korrelationsdifferenzen wurden die Korrelationskoeffizienten in Fishers Z-Werte (vgl. Cohen, 1988, S. 110) transformiert, da diese im Gegensatz zu Korrelationskoeffizienten annähernd normal verteilt sind (Bortz, 1999). Erst dadurch können die Werte subtrahiert werden.

Die berechneten Korrelationskoeffizienten zwischen der Mathematikangst und der Rechenleistung liegen teilweise über den Erwartungen. Dies ist möglicherweise darauf zurückzuführen, dass die vorliegende Stichprobe sowohl Kinder mit sehr geringen als auch sehr guten Rechenleistungen umfasst und sich die Stichprobe dadurch durch eine höhere Varianz auszeichnet. Wird beispielsweise allein die Gruppe der Kinder mit mindestens durchschnittlichen Rechenleistungen betrachtet, liegen die Korrelationskoeffizienten etwas darunter (MAI x HRT Addition: $r_{s}=-.192, p=072$, MAI x HRT Subtraktion: $r_{s}=-.278, p=$ .008 , MAI x RT BUEGA: $\left.r_{s}=-.294, p=.005\right)$ und demzufolge eher im Bereich bisheriger Untersuchungen. 


\section{Literatur}

Ashcraft, M. (2002). Math Anxiety: Personal, Educational, and Cognitive Consequences. Current Directions in Psychological Science, 11, 181-185.

Ashcraft, M. H. \& Faust, M. W. (1994). Mathematics Anxiety and Mental Arithmetic Performance: An Exploratory Investigation. Cognition and Emotion, 8, 97-125.

Ashcraft, M. H. \& Kirk, E. P. (2001). The relationships among working memory, math anxiety, and performance. Journal of Experimental Psychology: General, 130, 224-237.

Ashcraft, M. H., Krause, J. A., \& Hopko, D. R. (2007). Is math anxiety a mathematical learning disability? In D. B. Berch \& M. M. M. Mazzocco (Hrsg.), Why is math so hard for some children? (S. 329-348). Baltimore, MD: Brookes Publishing.

Ashcraft, M. H. \& Moore, A. M. (2009). Mathematics Anxiety and the Affective Drop in Performance. Journal of Psychoeducational Assessment, 27, 197-205.

Ashcraft, M. H., \& Ridley, K. S. (2005). Math anxiety and its cognitive consequences: A tutorial review. In J. I. D. Campbell (Hrsg.), Handbook of mathematical cognition (S. 315-327). New York: Psychology Press.

Baloğlu, M. \& Koçak, R. (2006). A multivariate investigation of the differences in mathematics anxiety. Personality and Individual Differences, 40, 1325-1335.

Bortz, J. (1999). Statistik für Sozialwissenschaftler (5. Aufl.). Berlin: Springer.

Bühner, M. (2006). Einführung in die Test- und Fragebogenkonstruktion (2. Aufl.). München: Springer.

Chinn, S. (2009). Mathematics anxiety in secondary students in England. Dyslexia, 15, 61-68.

Chipman, S. F., Krantz, D. H. \& Silver, R. (1992). Mathematics anxiety and science careers among able college women. Psychological Science, 3, 292-295.

Cohen, J. (1988). Statistical power analysis for the behavioral sciences. New Jersey: Lawrence Erlbaum Associates.

Devine, A., Fawcett, K., Szücs, D. \& Dowker, A. (2012). Gender differences in mathematics anxiety and the relation to mathematics performance while controlling for test anxiety. Behavioral and Brain Functions, 8, 1-9. 
Dew, K. M. H., Galassi, J. P. \& Galassi, M. D. (1984). Math Anxiety: Relation With Situational Test Anxiety, Performance, Physiological Arousal, and Math Avoidance Behavior. Journal of Counseling Psychology, 31, 580-583.

Dowker, A. (2005). Individual differences in arithmetic: Implications for psychology, neuroscience and education. Hove: Psychology Press.

Dowker, A., Bennett, K. \& Smith, L. (2012). Attitudes to mathematics in primary school children. Child Development Research. doi:10.1155/2012/124939

Döpfner, M., Schnabel, M., Goletz, H. \& Ollendick, T. (2006). Phobiefragebogen für Kinder und Jugendliche (PHOKI). Göttingen: Hogrefe.

Dreger, R. M. \& Aiken, L. R. (1957). The identification of number anxiety in a college population. Journal of Educational Psychology, 48, 344-351.

Esser, G., Wyschkon, A. \& Ballaschk, K. (2008). Basisdiagnostik Umschriebener Entwicklungsstörungen im Grundschulalter (BUEGA). Göttingen: Hogrefe.

Faust, M.W. (1992). Analysis of physiological reactivity in mathematics anxiety (Unveröffentlichte Doktorarbeit). Bowling Green State University: Bowling Green, Ohio.

Fehm, L. \& Fydrich, T. (2011). Prüfungsangst. Göttingen: Hogrefe.

Field, A. P. (2009). Discovering statistics using SPSS (and sex and drugs and rock'n'roll) (3. Aufl.). Newbury Park: Sage.

Haffner, J., Baro, K., Parzer, P. \& Resch, F. (2005). Heidelberger Rechentest. Göttingen: Hogrefe.

Hembree, R. (1990). The nature, effects, and relief of mathematics anxiety. Journal for Research in Mathematics Education, 21, 33-46.

Hopko, D. R., Ashcraft, M. H., Gute, J., Ruggiero, K. J. \& Lewis, C. (1998). Mathematics anxiety and working memory: Support for the existence of a deficient inhibition mechanism. Journal of Anxiety Disorders, 12, 343-355.

Hopko, D. R., Mahadevan, R., Bare, R. L. \& Hunt, M. K. (2003). The Abbreviated Math Anxiety Scale (AMAS): Construction, Validity, and Reliability. Assessment, 10, 178-183.

Hopko, D. R., McNeil, D. W., Zvolensky, M. J. \& Eifert, G. H. (2001). The relation between anxiety and skill in performance-based anxiety disorders: A behavioral formulation of social phobia. Behavior Therapy, 32, 185-207. 
Hyde, J. S., Fennema, E., \& Lamon, S. (1990). Gender differences in mathematics performance: A meta-analysis. Psychological Bulletin, 107, 139-155.

Imbo, I. \& Vandierendonck, A. (2007). The development of strategy use in elementary school children: Working memory and individual differences. Journal of Experimental Child Psychology, 96, 284-309.

Jain, S.\& Dowson, M. (2009). Mathematics anxiety as a function of multidimensional selfregulation and self-efficacy. Contemporary Educational Psychology, 34, 240-249.

Kamann, M. P. \& Wong, B. Y. (1994). Inducing adaptive coping self-statements in children with learning disabilities through self-instruction training. Journal of Learning Disabilities, $26,630-638$.

Kaufmann, L. \& von Aster, M. (2012). Diagnostik und Intervention bei Rechenstörung. Deutsches Ärzteblatt, 45, 767-780.

Krinzinger, H. \& Günther, T. (2013). Lesen, Schreiben, Rechnen - gibt es Unterschiede zwischen den Geschlechtern? Lernen und Lernstörungen, 2, 35-49.

Krinzinger H. \& Kaufmann, L. (2006). Rechenangst und Rechenleistung. Sprache, Stimme, Gehör, 30, 160-164.

Krinzinger, H., Kaufmann, L., Dowker, A., Thomas, G., Graf, M., Nuerk, H.-C. et al. (2007). Deutschsprachige Version des Fragebogens für Rechenangst (FRA) für 6- bis 9-jährige Kinder. Zeitschrift für Kinder- und Jugendpsychiatrie und Psychotherapie, 35, 341-351.

Krinzinger, H., Kaufmann, L. \& Willmes, K. (2009). Math anxiety and math ability in early primary school years. Journal of Psychoeducational Assessment, 27, 206-225.

Lambert, K. \& Spinath, B. (2012). Veränderungen psychischer Belastung durch Förderung von rechenschwachen Kindern und Jugendlichen. Zeitschrift für Kinder- und Jugendpsychiatrie und Psychotherapie, 41, 23-34.

Lang, P. J. (1968). Fear reduction and fear behaviour: Problems in treating a construct. In J. M. Schlien (Hrsg.), Research in Psychotherapy (3. Aufl., S. 90-102). Washington, DC: American Psychological Association.

Lyons, I. M. \& Beilock, S. L. (2012). When math hurts: Math anxiety predicts pain network activation in anticipation of doing math. PLoS One, 7, e48076. 
Ma, X. (1999). A meta-analysis of the relationship between anxiety towards mathematics and achievement in mathematics. Journal for Research in Mathematics Education, 30, 520540 .

Ma, X. \& Xu, J. (2004). The causal ordering of mathematics anxiety and mathematics achievement: a longitudinal panel analysis. Journal of Adolescence, 27, 165-179.

Maloney, E. A., Ansari, D. \& Fugelsang, J. A. (2011). The effect of mathematics anxiety on the processing of numerical magnitude. The Quarterly Journal of Experimental Psychology, 64, 10-16.

Maloney, E. A. \& Beilock, S. L. (2012). Math anxiety: who has it, why it develops, and how to guard against it. Trends in Cognitive Sciences, 16, 404-406.

Meece, J. L.,Wigfield, A., and Eccles, J. S. (1990). Predictors of math anxiety and its influence on young adolescents' course enrollment intentions and performance in mathematics. Journal of Educational Psychology, 82, 60-70.

Newstead, K. (1998). Aspects of children's mathematics anxiety. Educational Studies in Mathematics, 36, 53-71.

Pixner, S. \& Kaufmann, L. (2013). Prüfungsangst, Schulleistung und Lebensqualität bei Schülern. Lernen und Lernstörungen, 2, 111-124.

Punaro, L. \& Reeve, R. (2012). Relationships between 9-year-olds`math and literacy worries and academic abilities. Child Development Research. doi:10.1155/2012/359089

Ramirez, G., Gunderson, E. A., Levine, S. C. \& Beilock, S. L. (in press). Math anxiety, working memory and math achievement in early elementary school. Journal of Cognition and Development. doi: 10.1080/15248372.2012.664593

Richardson, F. C., \& Suinn, R. M. (1972). The Mathematics Anxiety Rating Scale: Psychometric data. Journal of Counseling Psychology, 19, 551-554.

Rubinsten, O. \& Tannock, R. (2010). Mathematics anxiety in children with developmental dyscalculia. Behavioral and Brain Functions, 46, 1-13.

Saß, H., Wittchen, H.-U. \& Zaudig, M. (1996). Diagnostisches und Statistisches Manual Psychischer Störungen (DSM-IV). Göttingen: Hogrefe.

Suinn, R. M., Taylor, S. \& Edwards, R. W. (1988). Suinn Mathematics Anxiety Rating Scale for Elementary School Students (MARS-E): Psychometric and Normative Data. Educational and Psychological Measurement, 48, 979-986. 
Thomas, G. \& Dowker, A. (2000). Mathematics anxiety and related factors in young children. Paper Presented at British Psychological Society Developmental Section Conference, Bristol.

Thurner, F. \& Tewes, U. (2000). Kinder-Angst-Test II (KAT-II). Göttingen: Hogrefe.

Vukovic, R. K., Kieffer, M. J., Bailey, S. P. \& Harari, R. R. (2013). Mathematics anxiety in young children: Concurrent and longitudinal associations with mathematical performance. Contemporary Educational Psychology, 38, 1-10.

Wood, G., Pinheiro-Chagas, P., Júlio-Costa, A., Micheli, L. R., Krinzinger, H., Kaufmann, L. et al. (2012). Math anxiety questionnaire: Similar latent structure in Brazilian and German school children. Child Development Research. doi:10.1155/2012/610192

Wu, S. S., Barth, M., Amin, H., Malcarne, V. \& Menon, V. (2012). Math anxiety in second and third graders and its relation to mathematics achievement. Frontiers in Psychology, 3, 1-11.

Young, C. B., Wu, S. S. \& Menon, V. (2012). The neurodevelopmental basis of math anxiety. Psychological Science, 23, 492-501.

Yüksel-Şahin, F. (2008). Mathematics anxiety among 4th and 5th grade Turkish elementary school students. International Electronic Journal of Mathematics Education, 3, 179-192. 


\section{Tabellen}

Tabelle 1: Stichprobenbeschreibung, Unterschiede zwischen Jungen $(n=47)$ und Mädchen $(n=89)$ in den Untersuchungsvariablen und im Alter, Ergebnisse der $t$-Tests

\begin{tabular}{|c|c|c|c|}
\hline Kognitive Leistung & $\begin{array}{r}\text { weiblich } \\
M(S D)\end{array}$ & $\begin{array}{r}\text { männlich } \\
M(S D)\end{array}$ & $\begin{array}{c}t \text {-Wert } \\
(d f=134)\end{array}$ \\
\hline Alter (in Monaten) & $99.35(8.69)$ & $101.13(10.40)$ & $-1.060, p=.291$ \\
\hline HRT Subtraktion ${ }^{1}$ & $42.26(9.15)$ & $44.68(12.20)$ & $\begin{array}{c}-1.195, p=.236 \\
(d f=73.99)\end{array}$ \\
\hline HRT Addition ${ }^{1}$ & $42.39(8.97)$ & 44.47 (10.99) & $\begin{array}{c}-1.113, p=.269 \\
(d f=78.92)\end{array}$ \\
\hline BUEGA Rechentest $^{1}$ & $46.69(11.95)$ & $48.21(11.60)$ & $-0.716, p=.475$ \\
\hline BUEGA Lesen gesamt $^{1}$ & $51.04(10.50)$ & $48.72(11.14)$ & $1.201, p=.232$ \\
\hline$\overline{\text { BUEGA Rechtschreibung }}^{1}$ & $48.97(11.32)$ & $43.89(10.45)$ & $2.550, p=.012$ \\
\hline BUEGA Gesamtintelligenz $^{1}$ & $52.74(8.50)$ & $48.72(8.04)$ & $2.670 p=.009$ \\
\hline${\mathrm{KAT}-\mathrm{II}^{1}}^{1}$ & $48.93(9.43)$ & $48.19(10.29)$ & $0.422, p=.674$ \\
\hline $\begin{array}{l}\text { Schul- und Leistungsangst } \\
\text { PHOKI }^{1}\end{array}$ & $53.99(10.17)$ & $54.26(10.11)$ & $-0.146, p=.884$ \\
\hline $\begin{array}{l}\text { Selbsteinschätzung und } \\
\text { Einstellung FRA }{ }^{1}\end{array}$ & $43.26(12.11)$ & $46.49(13.79)$ & $-1.410, p=.161$ \\
\hline
\end{tabular}

Anmerkung: ${ }^{1} \mathrm{~T}$-Werte 
Tabelle 2: Interkorrelationen des MAI, der Angstfragebögen und Rechenleistungen, Korrelationskoeffizienten nach Spearman $(\mathbf{n}=136)$

\begin{tabular}{|c|c|c|c|c|c|c|c|}
\hline Variable & $\begin{array}{c}\mathrm{M} \\
(\mathrm{SD})\end{array}$ & MAI & КАТ-II & PHOKI & FRA & $\begin{array}{c}\text { HRT } \\
\text { Addition }\end{array}$ & $\begin{array}{c}\text { HRT } \\
\text { Subtraktion }\end{array}$ \\
\hline 1. MAI & $\begin{array}{c}12.27 \\
(10.27)\end{array}$ & - & - & - & - & - & - \\
\hline 2. KAT-II & $\begin{array}{c}4.38 \\
(3.87) \\
\end{array}$ & $.697^{* * * *}$ & - & - & - & - & - \\
\hline 3. $\mathrm{PHOKI}(\mathrm{SL})$ & $\begin{array}{c}5.73 \\
(4.12) \\
\end{array}$ & $.702^{* * * *}$ & $.646^{* * *}$ & - & - & - & - \\
\hline 4. FRA & $\begin{array}{c}35.28 \\
(11.27)\end{array}$ & $-.656^{* * *}$ & $-.459^{* * *}$ & $-.532^{* * *}$ & - & - & - \\
\hline 5. HRT Addition ${ }^{1}$ & $\begin{array}{l}43.02 \\
(9.75) \\
\end{array}$ & $-.395^{* * *}$ & $-.243^{* *}$ & $-.169^{*}$ & $.529^{* * *}$ & - & - \\
\hline 6. HRT Subtraktion ${ }^{1}$ & $\begin{array}{c}43.03 \\
(10.32)\end{array}$ & $-.464^{* * *}$ & $-.236^{* *}$ & $-.253^{* *}$ & $.602^{* * *}$ & $.834^{* * * *}$ & - \\
\hline 7. Rechentest BUEGA ${ }^{1}$ & $\begin{array}{c}47.15 \\
(11.78)\end{array}$ & $-.419^{* * *}$ & $-.232^{* *}$ & $-.257^{* *}$ & $.525^{* * *}$ & $.581^{* * *}$ & $.705^{* * *}$ \\
\hline
\end{tabular}


Tabelle 3: Korrelationskoeffizienten für den Zusammenhang des MAI und der Angstfragebögen sowie der Rechenleistungen, Effektgrößenmaß $(q)$ und z-Werte für die Korrelationsdifferenz zwischen der Jungen- und der Mädchenstichprobe

\begin{tabular}{|c|c|c|c|c|}
\hline Variable & $\begin{array}{l}\text { männlich } \\
(n=47)\end{array}$ & $\begin{array}{l}\text { weiblich } \\
(n=89)\end{array}$ & $q$ & z-Wert \\
\hline KAT-II & $.752^{* * * *}$ & $.676^{* * * *}$ & 0.16 & 0.84 \\
\hline PHOKI & $.622^{* * *}$ & $.736^{* * *}$ & 0.21 & -1.15 \\
\hline FRA & $-.720^{* * *}$ & $-.606^{* * *}$ & 0.21 & 1.11 \\
\hline HRT Addition $^{1}$ & $-.471^{* *}$ & $-.355^{* *}$ & 0.15 & 0.76 \\
\hline HRT Subtraktion ${ }^{1}$ & $-.445^{* *}$ & $-.504^{* * *}$ & 0.08 & -0.41 \\
\hline Rechentest BUEGA $^{1}$ & $-.496^{* * *}$ & $-.356^{* *}$ & 0.17 & 0.93 \\
\hline
\end{tabular}

Anmerkung: ${ }^{1} \mathrm{~T}$-Werte, ${ }^{* * *} p<.001,{ }^{* *} p<.01$ 
Tabelle 4: Partialkorrelationskoeffizienten des MAI und der kognitiven Leistungen unter Kontrolle der Gesamtintelligenz sowie der allgemeinen Ängstlichkeit (KAT-II) und der Schul- und Leistungsangst (PHOKI)

\begin{tabular}{|c|c|c|c|c|c|}
\hline $\begin{array}{l}\text { Kontrollvariable } \\
\text { Gesamtintelligenz }\end{array}$ & Gesamt $(N=136)$ & männlich ( $n=47)$ & weiblich $(n=89)$ & $q$ & z-Wert \\
\hline HRT Addition $^{1}$ & $-.359^{* * * *}$ & $-.351^{*}$ & $-.332^{* * *}$ & 0.02 & 0.12 \\
\hline HRT Subtraktion $^{1}$ & $-.413^{* * *}$ & $-.322^{*}$ & $-.439^{* * *}$ & -0.14 & -0.74 \\
\hline Rechentest BUEGA ${ }^{1}$ & $-.353^{* * *}$ & $-.355^{*}$ & $-.339^{* *}$ & 0.02 & 0.10 \\
\hline Lesen Gesamt ${ }^{1}$ & $-.108^{\text {n.s. }}$ & $.041^{\text {n.s. }}$ & $-.182^{+}$ & -0.23 & -1.21 \\
\hline Rechtschreibung $^{1}$ & $-.172^{*}$ & $036^{\text {n.s. }}$ & $-.284^{*}$ & -0.33 & $-1.77^{*}$ \\
\hline \multicolumn{6}{|c|}{ Allgemeine Ängstlichkeit } \\
\hline HRT Addition ${ }^{1}$ & $-.302^{* * * *}$ & $-.360^{*}$ & $.263^{*}$ & -0.11 & -0.58 \\
\hline HRT Subtraktion $^{1}$ & $-.392^{* * *}$ & $-.358^{*}$ & $.423^{* * *}$ & 0.08 & 0.41 \\
\hline Rechentest BUEGA $^{1}$ & $-.321^{* * * *}$ & $-.382^{* *}$ & $.290^{* *}$ & -0.10 & -0.56 \\
\hline \multicolumn{6}{|c|}{ Schul- und Leistungsangst } \\
\hline HRT Addition $^{1}$ & $-.355^{* * *}$ & $-.434^{* *}$ & $.293^{* *}$ & -0.16 & -0.88 \\
\hline HRT Subtraktion $^{1}$ & $-.365^{* * *}$ & $-.389^{* *}$ & $.347^{* *}$ & -0.05 & -0.26 \\
\hline Rechentest BUEGA $^{1}$ & $-.307^{* * *}$ & $-.420^{* *}$ & $.233^{*}$ & -0.21 & -1.13 \\
\hline
\end{tabular}

